AN. MED. INTERNA (Madrid) Vol. 18, N. ${ }^{\circ} 10$, pp. 521-524, 2001

\title{
Neumotórax en pacientes infectados por el virus de inmunodeficiencia humana
}

\author{
C. MARTÍNEZ-VÁZQUEZ, M. SEIJAS, A. OCAMPO, A. LÓPEZ, I. OLIVEIRA, \\ B. SOPEÑA, J. DE LA FUENTE, S. FREITA
}

Unidad de Enfermedades Infecciosas. Servicio de Medicina Interna. Complejo

Hospitalario Xeral-Cíes. Universidad de Santiago de Compostela. Vigo

\author{
PNEUMOTHORAX IN HUMAN IMMUNODEFICIENCY VIRUS (HIV) \\ INFECTED PATIENTS
}

\section{RESUMEN}

Objetivo: Se describen con cierta frecuencia pacientes infectados por VIH, que desarrollan neumotórax. Esta complicación aparece fundamentalmente en el contexto de coinfección por Pneumocystis carinii (NPC), pero también asociada a profilaxis con pentamidina inhalada, coinfección por otros gérmenes y a maniobras traumáticas.

Método: Revisamos los aspectos más importantes de los neumotórax objetivados en nuestra población infectada por VIH a lo largo de ocho años, antes del inicio de terapia HAART generalizada.

Durante este tiempo 97 pacientes fueron diagnosticados de NPC de certeza o probable y 148 pacientes fueron sometidos a profilaxis con pentamidina inhalada mensual. Hemos recogido sólo 14 episodios de neumotórax en 13 pacientes. En diez ocasiones estuvieron en relación a maniobras agresivas, en tres a infección pulmonar concomitante, no estando en relación a patología pulmonar concreta en uno.

En cuanto a las maniobras agresivas consistieron en: cateterización de vena subclavia en seis (uno de estos tenía NPC de certeza y otro neumonía por neumococo), en un paciente tras biopsia transbronquial (también con NPC de certeza), en dos agresiones con arma blanca y en el último post-PAAF de adenopatía axilar.

Resultados: Las tres infecciones pulmonares concomitantes consistieron respectivamente en tres enfermos en: NPC de certeza (siendo este el único caso con antecedente de profilaxis con pentamidina inhalada), tuberculosis pulmonar activa e infección pulmonar por Pseudomonas a. Todos los pacientes, menos uno, fueron sometidos a drenaje pleural. Nueve se resolvieron tras aspiración. Dos de los tres pacientes con infección pulmonar concomitante, así como el espontáneo, necesitaron pleurodesis, siendo necesaria resección quirúrgica para la resolución del neumotórax asociado a tuberculosis. En el momento de concluir esta revisión habían fallecido seis pacientes (media 7 meses) entre los cuales estaban los tres con NPC de certeza en el momento del neumotórax, el intervenido por tuberculosis y el afecto de infección por Pseudomonas a, presentando todos ellos cifras de CD4 $<100$ en el momento del neumotórax, cifra que sólo se apreció en dos de los siete restantes.

Conclusiones: El neumotórax es frecuente en pacientes infectados por VIH con coinfección por NPC, pero puede verse durante otras coinfecciones y sobre todo por maniobras agresivas. El neumotórax supone un dato de mal pronóstico en la infección por VIH.

PALABRAS CLAVE: Neumotórax. VIH. Pneumocystis carinii.

\section{ABSTRACT}

Objective: Patients with HIV who develop pneumothorax have been previously described. Pneumocystis carinii pneumonia $(P C N)$ is the lea ding cause of this complication, but infection by other pulmonary micro organism, inhaled pentamidine therapy and lung invasive manoeuvres have also been associated with pneumothorax in HIV infected patients.

Method: We review the most relevant clinical aspects of pneumotho rax in HIV-infected persons, gathered in our hospital along eight years, before HAART therapy was started.

During this time, 97 patients with PCN were diagnosed and 148 patients received prophylaxis with inhaled pentamidine. Only 14 episo des of pneumothorax in 13 patients, were recorded. In ten occasions pneumothorax was related to pulmonary invasive manoeuvres, pulmo nary infections were found in three and was considered spontaneous in one.

The pulmonary invasive manoeuvres were: subclavia vein catheteri sation in six cases (one of them was diagnosed of proved PCN and the other has pneumococcal pneumonia); transbronchial biopsy in one patient (also with proved PCN), knife chest trauma in two cases and after fine needle aspiration of an axillary lymph node in one patient.

Results: The pulmonary infections associated with pneumothorax in three patients were: proved PCN (this patient was the only one in the group with inhaled pentamidine prophylaxis who developed pneumotho rax), active pulmonary infection by mycobacterium tuberculosis and Pseudomonas aeruginosa pneumonia. A drainage chest tube was placed in 12 patients with complete resolution in nine. In the other two patients pleurodesis was necessary and surgical repair was carried out in the other one (who had pulmonary tuberculosis). During the follow up six patients died (median time to death: 7 months). Among patients who died, five had pulmonary infections when the pneumothorax was diagno sed: PCN in three cases, pulmonary tuberculosis and pseudomonas pneumonia in the other two; all of them with less than 100 CD4 lymp hocytes.

Conclusions: Pneumothorax is frequent in HIV-infected patients with PCN, but other lung infections and, above all pulmonary invasive manoeuvres, can cause this complication. In our experience, HIV-infec ted patients who develop pneumothorax have a bad prognosis.

KEY WORDS: Pneumothorax. HIV Infection. Pneumocystis carinii.

Martínez-Vázquez C, Seijas M, Ocampo A, López A, Oliveira I, Sopeña B, de la Fuente J, Freita S. Neumotórax en pacientes infectados por el virus de inmunodeficiencia humana. An Med Interna (Madrid) 2001; 18: 521-524.

Trabajo aceptado: 16 de Mayo de 2001

Correspondencia: César Martínez Vázquez. C/ Gran Vía, 3, 2º 36204 . Vigo 


\section{INTRODUCCIÓN}

Se ha descrito en los últimos años la aparición de neumotórax en pacientes infectados por VIH, tanto en el contexto de coinfección por Pneumocystis carinii (NCP) (1-4), como en pacientes sometidos a profilaxis con pentamidina inhalada (5$7)$ o afectos de otras coinfecciones $(1,8,9)$. Pocos trabajos han destacado la importancia de neumotórax traumático en estos enfermos, sometidos con frecuencia a maniobras invasivas $(1,2,10)$. En cualquier caso parece que este evento es frecuente en este grupo de pacientes infectados por VIH $(11,12)$ y que su presencia conlleva mortalidad aumentada con respecto a los que no lo sufren $(1,5,10,13)$.

Revisamos a continuación los aspectos más importantes, tanto etiológicos como evolutivos, de los neumotórax objetivados en nuestra población infectada por VIH a lo largo de ocho años, antes de que se generalizara el uso del tratamiento antirretroviral muy activo (HAART).

\section{PACIENTES Y MÉTODOS}

Se revisan desde enero de 1989 a febrero de 1997, fecha en la que se generaliza en nuestro centro de terapia HAART, todos los pacientes controlados en nuestra Unidad de Enfermedades Infecciosas infectados por $\mathrm{VIH}$, que en algún momento de su evolución presentaron neumotórax. Se utilizó para dicha revisión los archivos computerizados de dicha unidad, contrastándose los enfermos recogidos con los registrados como neumotórax en la base de datos del Servicio de Cirugía Torácica desde 1991 hasta febrero 1997. Se revisan las historias clínicas de todos ellos, tabulándose en el momento del neumotórax los siguientes datos: cifra de CD4, enfermedades pasadas y concomitantes, técnicas invasivas o agresiones sufridas, datos demográficos, aspectos radiológicos y evolutivos con las distintas terapéuticas utilizadas y causa de, y tiempo transcurrido, hasta la muerte.

Se realizó el diagnóstico de certeza de NPC cuando mediante técnicas microbiológicas o histopatológicas se objetivó el diagnóstico, de probable NPC cuando existía un cuadro clínico y radiológico compatible y buena respuesta al cotrimoxazol o pentamidina E.V.

Se recogen también el número de pacientes sometidos a profilaxis con Pentamidina inhalada, aquellos que fueron diagnosticados de NPC de certeza o probable y tuberculosis pulmonar durante este tiempo en la unidad, así como la sobrevivencia media de los pacientes diagnosticados de NPC.

\section{RESULTADOS}

Desde enero de 1989 a febrero de 1997, se han controlado en nuestro centro 1150 pacientes infectados por VIH. Fueron sometidos a profilaxis con pentamidina inhalada mensual 148 pacientes, 97 pacientes fueron diagnosticados de neumonía de certeza o probable, por Pneumocystis carinii (NPC) y 160 de tuberculosis pulmonar. Durante este periodo han fallecido 303 pacientes en relación a patología relacionada con el VIH. El total de neumotórax atendidos por Cirugía Torácica fue de 299. Hemos recogido en esta serie de pacientes infectados por VIH sólo 14 episodios de neumotórax espontáneos o traumáticos en 13 pacientes, durante este periodo. Tres fueron diag- nosticados en 1991, cuatro en 1994, dos en 1995 y cinco en 1996. En diez ocasiones estuvieron en relación a maniobras agresivas traumáticas, en tres a infección pulmonar concomitante, no estando en relación con patología pulmonar alguna en un enfermo. Las maniobras invasivas consistieron en seis ocasiones en cateterización de una subclavia confirmándose en uno de ellos NPC concomitante y en otro neumonía por Neumococo; en otro paciente con NPC el neumotórax se produjo post-biopsia transbronquial, en otros dos ocurrió postagresión torácica con arma blanca y en el último post-punción-aspiración con aguja fina (PAAF) de adenopatía axilar. En cuanto a los tres casos de neumotórax no traumático en relación a infección pulmonar concomitante éstos consistieron en: un caso de NPC de certeza, otro tuberculosis pulmonar confirmada, (este último ya había padecido tuberculosis pulmonar 6 años antes) y otro infección por Pseudomonas a. Todos los pacientes salvo el del neumotórax post-PAAF de adenopatía axilar, fueron o estaban ingresados en el momento de producirse el neumotórax.

La media de edad en el momento de neumotórax fue de $35,0+/-4,11$. Todos salvo una mujer infectada por relación heterosexual fueron varones adictos a drogas por vía parenteral (ADVP). En ocho la cifra de CD4 estaba por debajo de 100 $\mathrm{xmm} 3$, estando incluido en este grupo los tres con NPC, el afecto de tuberculosis y el infectado por Pseudomonas y en los cinco pacientes restantes por encima de $400 \mathrm{xmm}^{3}$. Sólo se recogió el antecedente de profilaxis primaria con Pentamidina inhalada desde hacia tres años en el paciente con neumotórax espontáneo con NPC. Por otro lado, existía el antecedente, en un paciente con neumotórax post-subclavia y en el infectado por Pseudomonas, de NPC, de certeza, cuatro años y un año antes respectivamente. En los estudios radiológicos no se apreció neumotórax bilateral en ningún caso, ni tampoco predominio de hemitórax, obligando por su tamaño, salvo en un episodio (neumotórax post-PAAF), a la colocación de tubo de drenaje torácico. Se apreciaron imágenes quísticas subpleurales en un paciente con neumotórax secundario a colocación de subclavia con NPC activa y en el paciente con neumotórax no traumático con NPC. De los 12 neumotórax tratados con tubo de torascotomía y otro tratado con neumovent, nueve se resolvieron después de una media de 5 días de aspiración. El neumotórax sin patología pulmonar concomitante, el no traumático asociado a NPC y el asociado a infección por Pseudomonas necesitaron para su curación de pleurodesis química con tetraciclina el primero y talco los restantes. El secundario a tuberculosis a los 28 días de su ingreso y tras fracasar el tubo de toracostomía, fue sometido a toracotomía, al no encontrar cámara para retirar la videotoracoscopia, realizándose resección de lóbulo superior izquierdo cuyo estudio anátomo-patológico mostró datos de actividad tuberculosa. No se apreció recidiva, ni neumotórax contralateral en ningún caso. Estos datos quedan reseñados en la tabla I.

Hasta la fecha de tabulación de datos habían fallecido seis pacientes, de los cuales tres fueron los que tenían NPC de certeza durante el neumotórax, el cuarto el intervenido por tuberculosis, el quinto el infectado por Pseudomonas y el sexto uno de los traumáticos por cateterización de subclavia con antecedentes de NPC un año antes. Las causas de muerte y tiempo transcurrido hasta la misma, después del neumotórax, respectivamente, fueron: en uno de ellos cuatro meses más tarde por infección por Mycobacterium avium-complex, en tres con cuadro neurológico no filiado 1,6 y 17 meses más tarde res- 
TABLA I

DATOS CLÍNICOSY TERAPÉUTICOS DE LOS 13 PACIENTES CO N NEUM OTÓ RAX

\begin{tabular}{|c|c|c|c|c|c|c|}
\hline № de paciente & Edad & $\begin{array}{l}\text { Fecha diagnóstico } \\
\text { infección por VIH }\end{array}$ & $\begin{array}{l}\text { Fecha del } \\
\text { neumotórax }\end{array}$ & $\begin{array}{l}\text { Estadio } \\
- \text { CD4 + }\end{array}$ & $\begin{array}{l}\text { Etiología del } \\
\text { neumotórax }\end{array}$ & Tratamiento \\
\hline 1 & 30 & En. 91 & $1 / 08 / 94$ & C3 - 21 & N.P.C. & $\begin{array}{l}\text { T.T. } 7 \text { días } \\
\text { + pleurodesis } \\
\text { con talco }\end{array}$ \\
\hline 2 & 34 & 89 & 29/8/95 & C3 -70 & TB. & $\begin{aligned} & \text { T.T. } 28 \text { días } \\
& \text { + resección del LSI }\end{aligned}$ \\
\hline 3 & 41 & 87 & $14 / 11 / 96$ & C3 -28 & Vía central & N.V. 3 días \\
\hline 4 & 43 & En. 91 & $6 / 3 / 91$ & C3 -25 & N.P.C. +B.T. & T.T. 8 días \\
\hline 5 & 35 & 88 & $19 / 6 / 96$ & C3 -7 & Vía central & T.T. 6 días \\
\hline 6 & 39 & 94 & $21 / 11 / 96$ & C3 -24 & Vía central + N.P.C. & T.T. 6 días \\
\hline \multirow[t]{2}{*}{7} & 34 & 85 & 27/9/91 & A1 -671 & '91: arma blanca & T.T. 6 días \\
\hline & & & $20 / 10 / 96$ & A2 - 408 & '96: vía central & T.T. 6 días \\
\hline 8 & 39 & 87 & $27 / 7 / 94$ & B2 -469 & Espontáneo & $\begin{array}{c}\text { T.T. } 8 \text { días } \\
\text { + pleurodesis } \\
\text { con tetraciclinas }\end{array}$ \\
\hline 9 & 36 & 87 & $22 / 9 / 96$ & A1 -580 & $\begin{array}{l}\text { Vía central } \\
\text { Neumonía por } \\
\text { S. pneumonia }\end{array}$ & T.T. 2 días \\
\hline 10 & 29 & Jun. 87 & $7 / 11 / 94$ & C1 - 608 & $\begin{array}{c}\text { PAAF de } \\
\text { adenopatía axilar }\end{array}$ & No ingresó \\
\hline 11 & 35 & Nov. 90 & 22/3/91 & $A 2-450$ & Arma blanca & T.T. 6 días. \\
\hline 12 & 32 & 85 & $29 / 12 / 94$ & C3 -54 & $\begin{array}{l}\text { Neumonía por } \\
\text { P. aeruginosa }\end{array}$ & $\begin{array}{l}\text { T.T. } 4 \text { días } \\
\text { + pleurodesis } \\
\text { con talco }\end{array}$ \\
\hline 13 & 34 & En. 88 & $18 / 5 / 95$ & C3 - 6 & Vía central & T.T. 5 días \\
\hline
\end{tabular}

TB.: tuberculosis. N.P.C.: neumonía por Pneumocystis carinii. T.T.: tubo de toracostomía. B.T.: biopsia transbronquial. N.V.: Neumovent.

pectivamente, el quinto al cabo de 12 meses por enfermedad por citomegalovirus y el último un mes más tarde por insuficiencia respiratoria por bronconeumonía no filiada etiológicamente. La media de sobrevivencia de los pacientes diagnosticados de NPC en nuestro centro en las fechas en que se realizó el estudio era de 24,5 meses.

\section{DISCUSIÓN}

La frecuencia de neumotórax objetivada en nuestra población infectada por VIH, 14 de 1150 (1,2\%) y la de neumotórax en pacientes diagnosticados de NPC, 3 de 97 (3,09\%), resulta discretamente inferior a la apreciada en otras series descritas también como la muestra antes de terapia HAART. Así Sepkowitz et al (11) describen neumotórax de tipo traumático o por procesos pulmonares en un $2 \%$ de 1.030 pacientes con sida a lo largo de 9 años y Byrnes et al (14) observan porcentajes superiores $(4,35 \%)$ en una población de 574 pacientes con sida, observados durante tres años. Si tuviésemos en cuenta como hacen estos autores, sólo a pacientes con SIDA, nuestro porcentaje de neumotórax aumentaría. Con respecto a la frecuencia de neumotórax en NPC, Truitt et al (15) objetivan neumotórax en un $4 \%$ de sus pacientes con antecedentes de NPC y Colcer et al (13) sobre 154 pacientes con NPC un $6.5 \%$ de neumotórax.

La pentamidina inhalada, desde las primeras comunicaciones de neumotórax en pacientes infectados por VIH, ha sido imputada como causa predisponente por la pobre difusión alcanzada por el fármaco en vértices asociándose el neumotórax al desarrollo de NPC focal $(14,15)$. Sólo uno de nuestros 148 pacientes en programa de pentamidina inhalada desarrolló neumotórax. Aunque el antecedente del uso de pentamidina inhalada se sigue asociando a riesgo de neumotórax (4), otros autores, como nosotros, no encuentran ninguna relación entre los dos hechos (3), imputándose más bien a NPC concomitante como causante de neumotórax (18). Nuestro único enfermo con pentamidina y neumotórax, también tenía NPC. 
El mecanismo mediante el cual la NPC condicionaría neumotórax es también controvertido. Así Slabbynck et al (19), mediante videotoracoscopia, encuentran como causa desencadenante de neumotórax, en cuatro de cinco pacientes con NPC, pequeños a grandes nódulos subpleurales blanco-amarillentos dispersos en la superficie pulmonar. Estos autores (19) encuentran sólo en dos casos, y concomitantes a estas lesiones nodulares, quistes-bullas subpleurales, proponiendo tras estudio de piezas quirúrgicas de pulmón, al igual que otros (18), que el neumotórax está condicionado por enfermedad activa y destructiva pulmonar causada por el NPC. Otros, sin embargo imputan a la presencia de quistes-bullas presentes en pacientes con NPC activa o pasada, la aparición de neumotórax (3) (17). En nuestro único caso de neumotórax no traumático asociado a NPC se apreciaron quistes subpleurales en ambos lóbulos superiores.

Otro agente etiológico de neumotórax descrito en muy pocas ocasiones $(2,7,13,20,21)$ es el Mycobacterium $t$. Nuestro paciente afecto de tuberculosis y neumotórax requirió resección quirúrgica de la zona afectada, después de 26 días de aspiración mediante tubo de toracostomía y fracasar el intento de videotoracoscopia por adherencias pleurales. Este caso nos sitúa en una prevalencia de neumotórax en pacientes con tuberculosis pulmonar e infección por VIH $(0,62 \%)$ semejante a la descrita por otros autores en nuestro país (22).

Pocos trabajos describen la incidencia de neumotórax por maniobras invasivas en pacientes infectados por VIH $(1,2,10)$. En diez pacientes de nuestra serie, la etiología del neumotórax es imputable a esta causa, si bien como describen Ingran et al (10) en un alto porcentaje de estos pacientes se constató concomitantemente NPC, lo cual, y al margen de la maniobra invasiva, favorece sin duda alguna la aparición de neumotórax. Se ha descrito, por otro lado, claras diferencias evolutivas entre los neumotórax espontáneos y los producidos por maniobras invasivas, necesitando los primeros más tiempo de drenaje para su recuperación y con mayor frecuencia maniobras de esclerosis pleural o intervención quirúrgica (10).

Cuando a pesar del drenaje persiste pérdida aérea, situación frecuente en NPC, se ha propuesto primero esclerosis pleural con talco a poder ser con videotoracoscopia (19) (23), requiriéndose en muchas ocasiones ante el fracaso de esta medida intervención quirúrgica $(9,14,16,18,19)$. Incluso con esta medida la mortalidad del paciente con neumotórax, bien causada directamente o por otras causas distintas $(1,5,24)$, alcanza para varios autores el $50 \%$ de los pacientes $(9,13,20)$. Aunque en nuestra casuística no hubo fallecimientos atribuibles directamente al neumotórax, la sobrevivencia de los enfermos con antecedentes de NPC o NPC activa de nuestra serie de neumotórax, es claramente inferior a la apreciada en la población VIH con NPC pero sin neumotórax, por lo que el neumotórax asociado a PCP también a nosotros nos indicó un dato de mal pronóstico.

\section{Bibliografía}

1. Afessa B. Pleural effusion and pneumotorax in hospitalized patients with infection. The pulmonary complications, ICU support, and prognostic factors of hospitalized patients with HIV (PIP) study. Chest 2000; 117: 1031-1037.

2. McClean MD, Miller SB, Parsons PE, Cohn DL. Pneumothorax with Pneumocystis cariniin pneumonia in AIDS. Incidence and clinical characteristics. Chest 1991; 100: 1224-1228.

3. Coker R.J, Moss F, Peters B, et al. Pneumothorax in patients with AIDS. Resp Med 1993; 87: 43-47.

4. Metersky ML, Colt HG, Olson L.K, Shank TG. AIDS-related spontaneous pneumothorax. Risk factors and treatment. Chest 1995; 108: 946951.

5. Renzi PM, Corbeil C, Chassé M, et al. Bilateral pneumothorax hasten mortality in aerosolited pentamidine. Association with a lower dco prior to receiving aerosolized pentamidine. Chest 1992; 102: 491-496.

6. Martínez CM, Romanelli A, Mullen MP, et al. Spontaneous pneumothorax in AIDS patients receiving aerosolized pentamidine. Chest 1988; 94: $1317-1318$.

7. Leoung GS, Feigal DW Jr, Montgomery AB, et al. Aerosolized pentamidine for prophylaxis against Pneumocystis carinii pneumonia. N Engl J Med 1990; 323: 769-775.

8. Soubani AO. Pneumothorax during pulmonary tuberculosis un a HIVInfected patient. Chest 1993; 103: 1926-1927.

9. Leslie JM, Gallant JE, Chaisson RE. Pneumothorax in patients with AIDS. Infect Dis Clin Prac 1992; 1: 308-313.

10. Ingram RJ, Call S, Andrade A, et al. Management and outcome pneumothorax in patients infected with human inmunodeficiency virus. Clin Infect Dis 1996; 23: 634-627.

11. Sepkowitz KA, Telzak EE, Gold JW, et al. Pneumothorax in AIDS. Ann Intern Med 1991; 114: 455-459.

12. Coker RJ, Peters B, Mitchell D. Risk for pneumothorax in AIDS. Ann Intern Med 1991; 115: 157

13. Wait MA, Dal Nogare AR. Treatment of AIDS-Related spontaneous

pneumothorax. Chest 1994; 106: 693-696.

14. Byrnes TA, Brevig JK, Yeoh CB. Pneumothorax in patients with adquired inmunodeficiency syndrome. J Thorac Cardiovasc Surg 1989; 98 : 546-550.

15. Truitt T, Bagheri K, Safirstein BH. Spontaneous Pneumothorax in Pneumocystis carinii Pneumonia: Common or Uncommon? AJR 1992; 158: $916-917$

16. Scannell KA. Pneumothoraces and Pneumocystis carinii pneumonia in two AIDS patients receiving aerosolized pentamidine. Chest 1990; 97: $479-480$.

17. Stanley DJ, Luyclix BA, Haggerty MF, et al. Spontanous patients with recurrent Pneumocystis carinii pneumonia despiste aerosolized pentamidine prophylaxis. Chest 1991; 99: 502-504.

18. Beers MF, Sohn M, Swartz M. Recurrent pneumothorax in AIDS patients with Pneumocystis pneumonia. A clinicopatologic report of three cases and review of the literature. Chest 1990; 98: 266-270.

19. Slabbynck H, Kovitz KL, Vialette JP, et al. Thoracoscopic findings in spontaneous pneumothorax in AIDS. Chest 1994; 106: 1582-1586.

20. Tumbarello M, Tacconelli E, Pirronti T, et al. Pneumotorax in HIV infected patients: role of Pneumocystis carinii pneumonia and pulmonary tuberculosis. Eur Respir J 1997; 10: 1332-1335.

21. Rodríguez Arrondo F, Von Wichmann M, Arrizabalaga J, Iribarren JA, Idígoras P. Lesiones pulmonares cavitadas en los pacientes infectados por el virus de inmunodeficiencia humana: análisis de una serie de 78 casos. Med Clin (Barc) 1998; 111: 725-730.

22. Rivero A, López T, Santos J, et al. Neumotórax en paciente con SIDA Enferm Infecc Microbiol Clin 1994; 12: 452-454.

23. Kimmel RD, Karp MP, Cascona JJ, Zinno JS. Talc pleurodesis during video thoracoscopy for Pneumocystis carinii pneumonia -Related pneumothorax. A new technique. Chest 1994; 105: 314-315.

24. Azoulay E, Parrot A, Flahault A, et al. AIDS related Pneumocystis carinii pneumonia in the era of adjunctive steroids: implication of BAL neutrophilia. Am J Respir Crit Care Med 1999; 160: 493-499. 